\title{
Numerical simulation of shock-wave structure for argon and helium
}

\author{
Tatiana G. Elizarova \\ Institute of Mathematical Modeling, Russian Academy of Sciences, Miusskaya square 4a, \\ Moscow 125047, Russia \\ Ivan A. Shirokov \\ Faculty of Computing Mathematics and Cybernetics, M. V. Lomonosov Moscow State University, \\ Vorobjovy Gory, Moscow 119899, Russia \\ Salvador Montero \\ Instituto de Estructura de la Materia, CSIC, Serrano 121, Madrid 28006, Spain
}

(Received 21 September 2004; accepted 30 March 2005; published online 26 May 2005)

\begin{abstract}
We compare the thickness of shock-wave fronts at different Mach numbers, modeled via NavierStokes (NS) equations, with experimental results from the literature. Monoatomic argon and helium are considered. In this modeling a finite-difference scheme with second-order spatial accuracy is employed. For argon the calculated density thickness is in good agreement with the experimental results. For helium the NS results agree well with those from the bimodal model and with the few available experimental data. (C) 2005 American Institute of Physics. [DOI: 10.1063/1.1921267]
\end{abstract}

The strong gradients within a shock-wave lead to a number of associated effects of scientific and technological relevance which have attracted the attention of theoreticians and experimentalists for a long time. Though the main features of shock waves are well understood, much remains to be done to complete the prediction of their quantitative aspects. The structure of a stationary shock wave has often been employed as a testing problem for numerical models of rarefied gas flows. In this context the one-dimensional (1D) shock waves produced in atomic argon and helium have been usual test systems to check the numerical aspects of NavierStokes (NS) equations by comparison with experiment.

From the work done around 1950-1965 it was claimed that the NS approach could yield a reliable description of the density profile of the shock waves just up to Mach number $\mathrm{Ma} \sim 2$. This is more or less in accordance with the general validity criteria for the continuum description, as obtained from the classical Chapman-Enskog expansion of Boltzmann equation. A discussion of these aspects has been given by Kogan, ${ }^{1}$ Cercignani, ${ }^{2}$ and others. ${ }^{3-5}$ Moreover, due to the sparse experimental temperature and velocity data on welldefined 1D shock waves produced in shock tubes, little was known about the actual merits of NS equations to model the profiles of these quantities across the shock wave. This limitation has prevailed up to now in spite of the wealth of density and temperature data on $2 \mathrm{D}$ shock waves produced in jets. ${ }^{6,7}$ Unfortunately, 2D flows pose a number of difficulties for numerical modeling of normal shock waves (complicated flow pattern, low temperature upstream of the shock, with poorly known dependence of the viscosity on the temperature). Therefore, 2D shock waves are not well suited for use in connection with the simple 1D mathematical formulation.

The main source of experimental 1D shock-wave density data suited for the present purpose has been compiled and completed with accurate original data by Alsmeyer. ${ }^{8}$ In this work the experimental reciprocal thickness of argon and helium 1D shock waves is shown to be well characterized up to
$\mathrm{Ma}=10$, while a few sparse helium data points have been included. Complete argon experimental density profiles were also reported by Alsmeyer for several $\mathrm{Ma}<10$ values. Due to the shortage of experimental data on helium, the results of bimodal calculations have been also used as a reference, ${ }^{9}$ since they are known to be quite reliable for shock-wave modeling. ${ }^{10}$

In the present work the calculations of 1D shock-wave structures have been carried out by employing the NS equations, the results being compared with the referred experimental data ${ }^{8}$ and bimodal model. ${ }^{9,10}$ Neither the velocity nor the temperature profiles were measured in the former experiments. The temperature upstream of the shock wave was $300 \mathrm{~K}^{8}$, allowing us to employ a simple and well founded description of the thermal dependence of the viscosity by means of the parameters reported by Bird. ${ }^{11}$

The NS equations for the one-dimensional plane flow read

$$
\begin{aligned}
& \frac{\partial \rho}{\partial t}+\frac{\partial j}{\partial x}=0, \\
& \frac{\partial(\rho u)}{\partial t}+\frac{\partial(j u)}{\partial x}+\frac{\partial p}{\partial x}=\frac{\partial \Pi_{x x}}{\partial x}, \\
& \frac{\partial E}{\partial t}+\frac{\partial(j H)}{\partial x}+\frac{\partial q}{\partial x}=\frac{\partial\left(\Pi_{x x} u\right)}{\partial x} .
\end{aligned}
$$

Here $\rho$ stands for the gas density, $u$ for the velocity, $p$ $=\rho R T$ for the pressure, $T$ for the temperature, $\gamma$ for the specific heat ratio, and $R$ for the gas constant; $E=\rho u^{2} / 2+p /(\gamma$ $-1)$ and $H=(E+p) / \rho$ are, respectively, the total energy and enthalpy per unit volume. The mass flux density vector is $j$ $=\rho u$. The $x x$ component of the shear-stress tensor in Eqs. (2) and (3) is given by 


$$
\Pi_{x x}=\frac{4}{3} \eta \frac{\partial u}{\partial x}
$$

The heat flux vector is

$$
q=-\kappa \frac{\partial T}{\partial x}
$$

Viscosity coefficient $\eta$ and heat conductivity coefficient $\kappa$ are connected by the relations $\eta=\eta_{\infty}\left(T / T_{\infty}\right)^{\omega}, \quad \kappa$ $=\gamma R \eta /[(\gamma-1) \operatorname{Pr}]$, where $\eta_{\infty}$ is the value of $\eta$ for the temperature $T_{\infty}$ and Pr is Prandtl number.

For the numerical solution of the system (1)-(3) the computational domain is covered with a uniform computational grid with the space step $h_{x}$ and the time step $h_{t}$. The space derivatives are approximated by the central differences of the second order. The space step is taken much smaller than the shock wave thickness, ensuring the stability of the numerical algorithm without resorting to any artificial dissipation. The time derivatives are approximated by forward differences of the first order. The finite-difference scheme for the initial-boundary problem (1)-(3) is solved by means of an explicit algorithm where the steady-state solution is attained as the limit of a time-evolving process. The numerical solution is supposed to be achieved according to the criterium $\max (\hat{\rho}-\rho) / h_{t}<\varepsilon=10^{-3}$, where $\hat{\rho}$ and $\rho$ are the density values for consecutive time steps.

We calculate the shock-wave structure for Mach numbers in the range 1.5-10. Dimensionless quantities are introduced on the basis of the upstream gas parameters. The initial conditions are the following: for $x<0, \rho=\rho^{(1)}=1, u$ $=u^{(1)}=\mathrm{Ma}, p=p^{(1)}=1 / \gamma$, and for $x>0$, the values of $\rho^{(2)}$, $u^{(2)}, p^{(2)}$ are defined via the Rankine-Hugoniot conditions. ${ }^{12}$ The values of the upstream and downstream boundaries are fixed.

The reciprocal shock thickness is defined as $\lambda / \delta$, where $\lambda$ is the upstream mean-free path and $\delta$ is the shock thickness, calculated in dimensionless form by the maximum value of the finite-difference derivative $\partial \rho / \partial x: \lambda / \delta$ $=\max _{i}\left[\left(\rho_{i+1}-\rho_{i-1}\right) / 2 h_{x}\right] /\left(\rho^{(2)}-\rho^{(1)}\right)$.

According to Bird ${ }^{11}$ the upstream mean-free path is calculated as $\lambda=\eta /(\rho \sqrt{2 \pi R T} \Omega / 4)$, where $\Omega=30 /[(7-2 \omega)(5$ $-2 \omega)]$.

The following dimensionless finite-difference scheme is used:

$$
\begin{aligned}
\hat{\rho}_{i}= & \rho_{i}-\frac{h_{t}}{h_{x}}\left(j_{i+1 / 2}-j_{i-1 / 2}\right), \\
\hat{\rho}_{i} \hat{u}_{i}= & \rho_{i} u_{i}+\frac{h_{t}}{h_{x}}\left[\left(\Pi_{i+1 / 2}-\Pi_{i-1 / 2}\right)\right. \\
& \left.-\left(j_{i+1 / 2} u_{i+1 / 2}-j_{i-1 / 2} u_{i-1 / 2}\right)-\left(p_{i+1 / 2}-p_{i-1 / 2}\right)\right], \\
\hat{E}_{i}= & E_{i}+\frac{h_{t}}{h_{x}}\left[\left(\Pi_{i+1 / 2} u_{i+1 / 2}-\Pi_{i-1 / 2} u_{i-1 / 2}\right)\right. \\
& \left.\quad-\left(j_{i+1 / 2} H_{i+1 / 2}-j_{i-1 / 2} H_{i-1 / 2}\right)-\left(q_{i+1 / 2}-q_{i-1 / 2}\right)\right], \\
\hat{p}_{i}= & (\gamma-1)\left[\hat{E}_{i}-\hat{\rho}_{i}\left(\hat{u}_{i}\right)^{2} / 2\right],
\end{aligned}
$$

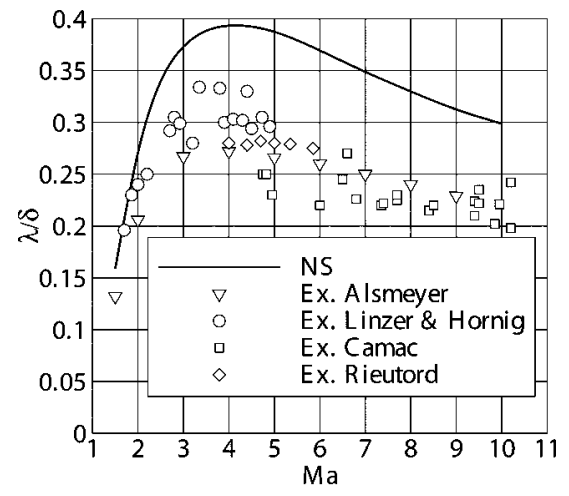

FIG. 1. Reciprocal shock wave thickness vs Mach number in argon.

$$
\begin{aligned}
& j_{i+1 / 2}=\left(\rho_{i+1} u_{i+1}+\rho_{i} u_{i}\right) / 2, \\
& \Pi_{i+1 / 2}=\frac{4}{3} \frac{1}{2}\left(\eta_{i+1}+\eta_{i}\right)\left(u_{i+1}-u_{i}\right) / h_{x}, \\
& q_{i+1 / 2}=-\frac{1}{(\gamma-1) \operatorname{Pr}} \frac{1}{2}\left(\eta_{i+1}+\eta_{i}\right)\left(T_{i+1}-T_{i}\right) / h_{x}, \\
& T_{i}=\gamma p_{i} / \rho_{i}, \quad E_{i}=\rho_{i} u_{i}^{2} / 2+p_{i} /(\gamma-1), \\
& \rho_{i+1 / 2}=\left(\rho_{i+1}+\rho_{i}\right) / 2, \quad u_{i+1 / 2}=\left(u_{i+1}+u_{i}\right) / 2, \\
& p_{i+1 / 2}=\left(p_{i+1}+p_{i}\right) / 2, \\
& E_{i+1 / 2}=\left(E_{i+1}+E_{i}\right) / 2, \quad H_{i+1 / 2}=\left(E_{i+1 / 2}+p_{i+1 / 2}\right) / \rho_{i+1 / 2} .
\end{aligned}
$$

The physical parameters were taken in the work of Bird: ${ }^{11}$ for argon- $\gamma=5 / 3, \omega=0.81, \mathrm{Sc}=0.752, \operatorname{Pr}=2 / 3$; for helium $-\gamma=5 / 3, \omega=0.66, \mathrm{Sc}=0.7575, \mathrm{Pr}=2 / 3$. The number of space grid points is $N_{x}=1200$, while the space step is $h_{x}$ $=0.25$. The time step is defined as $h_{t}=\alpha h_{x} / \max (\sqrt{T}+|u|)$, $\alpha=0.001$.

The calculated reciprocal shock thickness for argon is shown in Fig. 1 together with the experimental data collected by Alsmeyer. ${ }^{8}$ Alsmeyer's original results are marked by $\boldsymbol{\nabla}$. The experimental data were normalized for the hard sphere mean-free path $(\omega=0.5)$. This implies the following renormalization for our results: $\lambda(0.5)=1.32 \lambda(0.81)$ for argon; $\lambda(0.5)=1.16 \lambda(0.66)$ for helium.

Figure 2 shows the density, temperature, and velocity profiles in a $\mathrm{Ma}=9$ shock wave in argon. The density profiles shown are normalized as $f_{\rho}=\left(\rho-\rho^{(1)}\right) /\left(\rho^{(2)}-\rho^{(1)}\right)$, where $f_{\rho}$ is the value shown and $\rho^{(1)}, \rho^{(2)}$ are the boundary values. A similar normalization holds for the temperature. The normalization for the velocity is given by $f_{u}=\left(u-u^{(2)}\right) /\left(u^{(1)}-u^{(2)}\right)$. Figure 2 shows that the NS numerical results match reasonably Alsmeyer's density profile. ${ }^{8}$

In Fig. 3 the density profile of a $\mathrm{Ma}=9$ argon shock wave is depicted in some detail. The computational points, marked by dots, show the noticeable fluctuations of the numerical solution behind the shock wave. These fluctuations display the periodicity of the spatial grid and are responsible for the slow convergence of the NS numerical method. 


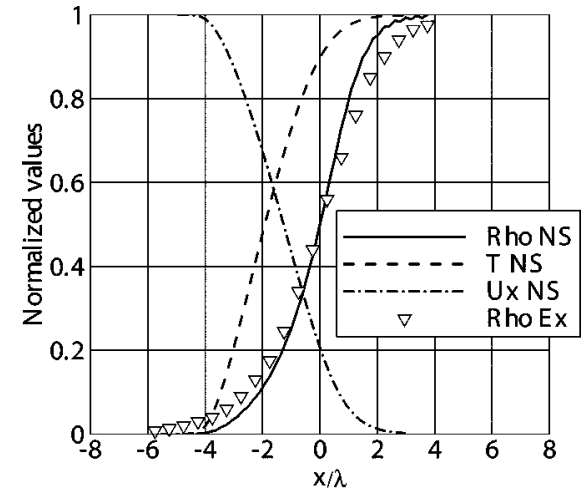

FIG. 2. Density, velocity, and temperature profiles in argon shock wave for $\mathrm{Ma}=9$.

The numerical algorithm was tested for grid convergence. For this purpose the NS-based modeling of a $\mathrm{Ma}=4$ argon shock wave with step $h_{x}=0.25, N_{x}=1200$, and $\alpha$ $=0.001$ was employed as a reference, yielding a reciprocal width $\lambda / \delta=0.297951$. For this variant the number of time steps to convergence is $N_{t}=4.73352 \times 10^{6}$. Using a double space step $h_{x}=0.5$, for $N_{x}=600$, and the same time step, we obtained $\lambda / \delta=0.290905\left(N_{t}=2.69736 \times 10^{6}\right)$. Using half the space grid size $h_{x}=0.125$, for $N_{x}=2400$, and a smaller time step derived from $\alpha=0.0001$, we obtained $\lambda / \delta$ $=0.302864\left(N_{t}=9.40607 \times 10^{7}\right)$. Both extreme values are close enough to the 0.297951 reference value. This assures a converged result and shows how weakly this result depends, within given limits, on grid and time step.

The results for helium are presented in Fig. 4, jointly with the few available experimental data ${ }^{8}$ and with the results from the bimodal approach. ${ }^{9}$ The bimodal approach, which is an approximate solution of the Boltzmann equation, is known to give an accurate description of shock-wave structure for high Mach numbers. The present results match reasonably well the experimental data for $\mathrm{Ma}=3$ and $\mathrm{Ma}$ $=4$, as well as the bimodal calculation at higher Ma.

In this work it is concluded that the Navier-Stokes calculated density profiles and reciprocal shock thickness in argon agree with the experiments ${ }^{8}$ much better than in previous works. ${ }^{1-5}$ The reciprocal shock thickness in helium agrees

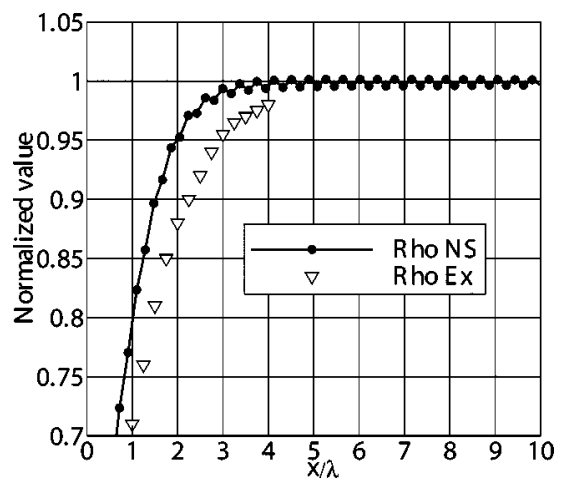

FIG. 3. Density profile in argon shock wave for $\mathrm{Ma}=9$ in detail.

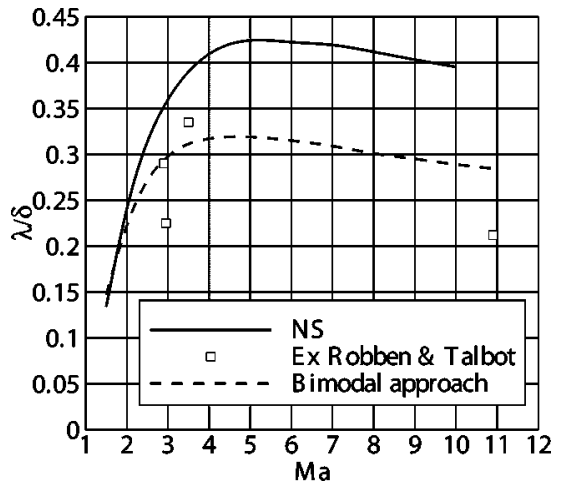

FIG. 4. Reciprocal shock-wave thickness vs Mach number in helium.

reasonably well with the results of bimodal calculations ${ }^{9}$ and with the few available experimental data.

The NS calculations were performed employing the second-order space approximation on a uniform spatial grid without artificial dissipation to stabilize the numerical solution. The numerical results from NS equations show a slow numerical convergence. This is related to the numerical oscillations of the solution. Such effect delays a converged NS solution, especially for large Mach numbers. The number of time steps for convergence varies in a range of $10^{5}-10^{8}$.

As shown here, the NS equations prove to be more precise than thought before for describing the density profile in an argon shock wave at Mach numbers in the range 1.5 $<\mathrm{Ma}<10$. The previously anticipated limitations of NS equations for the shock structure problems at large Mach numbers may be attributed to numerical problems and imperfections of earlier calculations. For instance, in Refs. 1-5 the NS results for argon density thickness are off by $\sim 100 \%$ at $\mathrm{Ma} \sim 6$

The authors are thankful to Professor T. Ytrehus for the fruitful discussions.

${ }^{1}$ M. N. Kogan, Rarefied Gas Dynamics (Plenum, New York, 1969).

${ }^{2}$ C. Cercignani, Rarefied Gas Dynamics (Cambridge University Press, Cambridge, 2000).

${ }^{3} \mathrm{~F}$. Robben and L. Talbot, "Measurements of shock wave thickness by the electron beam fluorescence method," Phys. Fluids 9, 633 (1966).

${ }^{4}$ K. Toba and J. D. Melnik, "Two-fluid model for shock wave structure," Phys. Fluids 8, 2153 (1965).

${ }^{5}$ M. Linzer and D. F. Hornig, "Structure of shock fronts in argon and nitrogen," Phys. Fluids 6, 1661 (1963).

${ }^{6}$ A. Ramos, B. Maté, G. Tejeda, J. M. Fernández, and S. Montero, "Raman spectroscopy of shock waves," Phys. Rev. E 62, 4940 (2000).

${ }^{7}$ I. A. Graur, T. G. Elizarova, A. Ramos, G. Tejeda, J. M. Fernández, and S. Montero, "Study of shock waves in expanding flows on the basis of spectroscopic experiments and quasi-gasdynamic equations," J. Fluid Mech. 504, 239 (2004).

${ }^{8} \mathrm{H}$. Alsmeyer, "Density profiles in argon and nitrogen shock waves measured by the absorption of an electron beam," J. Fluid Mech. 74, 497 (1976).

${ }^{9} \mathrm{C}$. Muckenfuss, "Some aspects of shock structure according to bimodal model," Phys. Fluids 5, 1325 (1962).

${ }^{10} \mathrm{H}$. Mott-Smith, "The solution of the Boltzmann equation for a shock wave," Phys. Rev. 82, 885 (1951).

${ }^{11}$ G. A. Bird, Molecular Gas Dynamics and the Direct Simulation of Gas Flows (Clarendon, Oxford, 1998).

${ }^{12}$ L. D. Landau and E. M. Lifshitz, Hydrodynamics (Nauka, Moscow, 1986). 